\title{
Grundlagenuntersuchung zu ausgewählten finiten Elementen der Strukturmechanik für ein einfaches validierbares FE-Modell
}

\author{
Norbert Miersch*, Robert Roßmann, Christian Holz
}

\section{Zusammenfassung}

Das Thema beschäftigt sich mit der Finiten-ElementeAnalyse (FEA) ausgewählter Elemente der Strukturmechanik für ein einfaches validierbares FE-Modell. In einer Grundlagenuntersuchung wird zunächst am Modell eines einseitig eingespannten Trägers das Konvergenzverhalten der Biegespannung und Verformung bezüglich ausgewählter Elemente überprüft. In einem weiteren Schritt wird der Einfluss der Art und Anzahl der Elemente und Knoten in Bezug zur Berechnungszeit für die Analyse statischer und dynamischer Problemstellungen untersucht und ausgewertet. Darüber hinaus wird der Einfluss der zeitlichen Auflösung (Inkrementierung) und der Elementqualität mit Rücksicht auf die Berechnungszeit analysiert. Es ist der Untersuchung zu entnehmen, dass das Konvergenzverhalten der Biegespannung und deren Verwendung aufgrund von Singularitäten gesondert zu betrachten ist. Im Ergebnis der Analyse wurde festgestellt, dass die Berechnungszeit mit der Zunahme von Elementen und Knoten, aber auch mit feiner werdender Zeitauflösung bei Anwendung einer dynamischen Analyse ansteigt.

\section{Abstract}

This research focuses on the analysis of selected elements of the finite element analysis of simple validated FE models. It shows the types of elements of the finite element method and their application, where the convergence behaviour of the bending stress and deformation of a cantilever beam under force loading relating to selected elements is represented. In addition, the number of elements and nodes related to the dynamic and static calculation time are investigated. Moreover, the influence of the time interval resolution and the element quality is analysed in consideration of the calculation time. One of the outcomes of this investigation is the significant behaviour of the bending stress in terms of the fixing and their resultant singularities. The calculation time increases with the growing number of elements and nodes as well as with a capillary time resolution related to the dynamic examination.

\section{Einleitung}

Die Anwendung der Finiten-ElementeMethode (FEM) ist Stand der Technik und hat sich in der industriellen Praxis als zweckmäßiges Werkzeug durchgesetzt. Sie ist demzufolge auch Bestandteil der studentischen Ausbildung im Studiengang Maschinenbau an der TH Wildau. Bezogen auf die traditionelle Maschinenbaubranche hat sich der Einsatz der Rechentechnik in den letzten Jahren besonders progressiv entwickelt. Simulationsverfahren, wie die FEM sind von besonderer Bedeutung, um die Anzahl der Versuche zu reduzieren und damit Entwicklungskosten und -zeit zu sparen.

Da es sich bei der FEM um ein Näherungsverfahren handelt, sind deren Ergebnisse, um Fehler beurteilen zu können, möglichst zu verifizieren. Für jede Simulation wird ein FE-Modell generiert, das mehr oder weniger dem realen Bauteil unter realen Bedingungen angenähert ist. Dabei wird ein Fehler in bestimmten Grenzen von 3 bis $5 \%$ toleriert. Hierzu können unterschiedliche „Finite Elemente“ zur Lösung ein und desselben Problems in die Simulation eingebunden werden. Eine zweckmäßige Modellbildung steht im direkten Zusammenhang mit der Wahl der finiten Elemente und kann bei komplizierten Bauteilen und/ oder großen Modellen erhebliche Rechenzeit sparen.

Interessant sind zunächst einmal grundlegende Untersuchungen zur Elementgenauigkeit. Deshalb wird in einem ersten Schritt auf die Genauigkeit von ausgewählten finiten Elementen bezogen auf ein einfaches validierbares FE-Modell eingegangen, ohne einen kostspieligen Versuchsaufwand zu betreiben.

\section{Einführung in die Problematik}

Die FEM ist ein Teilgebiet der numerischen Lösungsverfahren und wird als 
Simulationswerkzeug sowohl in der Entwicklung als auch in der Fertigung eines Produktes eingesetzt. Beispielhaft sind die Anwendung der strukturmechanischen Simulation während der Entwicklung/Konstruktion eines Produktes und die Nutzung der Erstarrungssimulation bei der Gussteilherstellung zu nennen (siehe Abb. 1).

Auch im späteren Service, wenn das Produkt im Alltag des Kunden genutzt wird und es möglicherweise zum Versagen kommt, sind Nachrechnungen bestimmter Betriebslasten und deren Auswirkungen auf die Konstruktion möglich. In diesem Szenario dient die FEM zur Ursachenanalyse. Zu Beginn einer jeden FEM-Untersuchung steht die Modellbildung. Hierbei wird das reale Bauteil einer Idealisierung zu einem vereinfachten Modell und einer Diskretisierung unterzogen. In diesem Schritt werden auch Randbedingungen berücksichtigt, welche beispielsweise Lagerungen, Kräfte und Momente definieren.

Als räumliche Diskretisierung wird das Zerlegen des Bauteiles in endlich kleine „finite Elemente“ bezeichnet (Klein 2015), wobei das Element selbst durch seine Randknoten definiert wird. Mit den finiten Elementen wird das Bauteil vernetzt. Bei der zeitlichen Diskretisierung werden die Simulationsergebnisse einem jeweiligen Zeitschritt zugeordnet, was bei dynamischen Berechnungen der Fall ist und erheblich mehr Rechenzeit erfordert.

Die nachstehende Tab. 1 zeigt vereinfacht einige Elementtypen und deren Verwendung (Wittel 2010).

In der nächsten Phase erfolgt die Aufstellung von Algorithmen, die Steifigkeitsmatrizen der Elemente zur Gesamtsteifigkeitsmatrix zusammenfassen. Aus diesen resultieren schließlich Ergebnisse wie Verformungen, Spannungen oder Auflagerreaktionen. Betrachtet man an dieser Stelle das Vorgehen der FEM, so erfolgt die mathematische Näherung der Lösung an den Gaußpunkten (Integrationspunkten) eines Elementes. Die Spannungen bzw. Dehnungen werden auf die Knoten des jeweiligen Elementes extrapoliert.

Die nachstehende Abb. 2 zeigt die Näherung an die Referenzfunktion über den Linearansatz bzw. über den

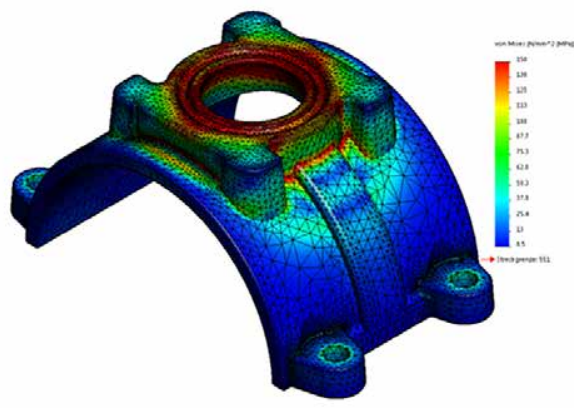

a) Ergebnisse (Vergleichspannungen) einer strukturmechanischen Simulation mittels FEM

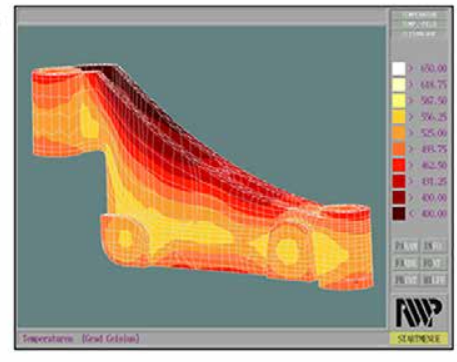

b) Ergebnisse (Temperaturfeld) eine Gießsimulation mittels FEM (Todte at al. 1999)

Abb. 1) Beispiele für Simulationsergebnisse aus a) Entwicklung/Konstruktion und b) Fertigung.

Polynomansatz. Die Referenzfunktion spiegelt in diesem Fall die Geometrie eines Bauteils wieder.

In Abb. 2 wird ersichtlich, dass zur Näherung über den Linearansatz eine deutlich höhere Anzahl an Elementen benötigt wird. Bei Verwendung eines Polynomansatzes kann eine bessere Annäherung mit wenigen Elementen erreicht werden. Dazu gehören beispielsweise quadratische oder kubische Funktionen, welche sich gut als Näherungs- bzw. Formfunktion für das finite Element eignen (Klein 2015). Die nachfolgenden Untersuchungen stützen sich im Wesentlichen darauf, mit welchen Elementen und welcher Elementanzahl eine Zielgröße, beispielsweise eine bestimmte Biegespannung, errechnet wird. Nähert man sich der Zielgröße, etwa einem theoretischen Wert, mit einer gewissen Elementanzahl an oder erreicht diesen Wert, liegt ein konvergentes Verhalten vor.

Die Konvergenz beschreibt die Annäherung an das physikalisch richtige Ergeb-

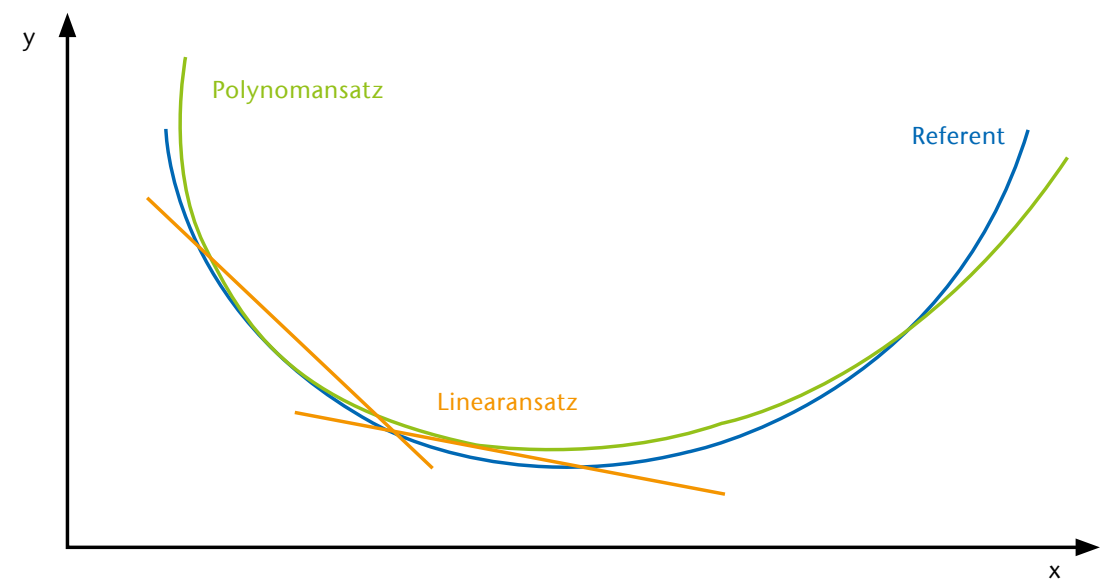

Abb. 2) Linearansatz und Polynomansatz. nis in Abhängigkeit vonder Netzdichte. Die Divergenz beinhaltet das deutliche Ansteigen des Ergebnisses in Bezug zur Netzdichte, welche bei Spannungen an scharfkantigen Kerben oder Einspannungen resultieren kann. Hier kann auch von Singularitäten gesprochen werden (Gebhardt 2014).

Die Validierung solcher FE-Modelle erfolgt beispielsweise mit theoretischen Überschlagsrechnungen, sofern dies im Hinblick auf die Bauteilkomplexität möglich ist. Mit ersten Prototypen können schließlich Testdaten generiert werden, welche dem Vergleich der FEModelle dienen. Man spricht an dieser Stelle von einer Verifizierung.

\section{Eingrenzungen}

Um Aussagen zur Genauigkeit und zum Konvergenzverhalten der verschiedenen finiten Elemente machen zu können, wird zunächst auf ein einfaches Balkenmodell eingegangen, dessen Ergebnisse man direkt den Ergebnissen einer analytischen Be- 
Tab. 1: Elementtypen und deren Verwendung

\begin{tabular}{|c|c|c|c|c|}
\hline Elementklasse & Dimension & Elementtyp & Anwendungsgebiet & Elementgeometrie \\
\hline Linienelemente & $1 \mathrm{D}$ & $\begin{array}{l}\text { Stab } \\
\text { Balken } \\
\text { Rohr } \\
\text { Feder } \\
\text { Dämpfer }\end{array}$ & $\begin{array}{l}\text { Fachwerke } \\
\text { Wellen } \\
\text { Rahmentragwerke } \\
\text { Rohrleitungen }\end{array}$ & Stab/Balken \\
\hline Flächenelemente & $2 \mathrm{D}$ & $\begin{array}{l}\text { Dreieck } \\
\text { Viereck } \\
\text { Scheibe } \\
\text { Platte } \\
\text { Schalen }\end{array}$ & $\begin{array}{l}\text { Blechbauteile } \\
\text { Karosseriebau } \\
\text { Behälter } \\
\text { Gehäuse } \\
\text { Rohrleitungen }\end{array}$ & Dreieck \\
\hline Volumenelemente & $3 \mathrm{D}$ & $\begin{array}{l}\text { Tetraeder } \\
\text { Hexaeder } \\
\text { Pentaeder }\end{array}$ & $\begin{array}{l}\text { Halter } \\
\text { Dicke Bauteile } \\
\text { Auflager }\end{array}$ & Tetraeder \\
\hline Sonstige Elemente & & $\begin{array}{l}\text { Federn } \\
\text { Dämpfer }\end{array}$ & $\begin{array}{l}\text { Randbedingungen } \\
\text { Hilfselemente }\end{array}$ & Dämpfer \\
\hline
\end{tabular}

trachtung mit Hilfe der technischen Mechanik gegenüberstellen kann. Die Ergebnisse können dann für weitere Untersuchungen von komplexeren Bauteilen bzw. Baugruppen herangezogen werden. Die Simulation wird mit dem FEM-Programm ANSYS Workbench durchgeführt.

Der Umfang der Analyse wird eingegrenzt auf Untersuchungen zu Dreiecks- und Viereckelementen sowie zu den Volumenelementen als Tetraeder, Hexaeder und Prisma. Für alle genannten finiten Elemente wird der lineare und quadratische Näherungsansatz untersucht (mit und ohne Zwischenkoten). Die Validierung und Prüfung der Plausibilität der Ergebnisse basiert auf dem Ansatz des Timoshenko-Balkens (Berücksichtigung der Biege- und Schubverformung). Für die Belastung wurden zwei Lastfälle vorgesehen. Die Belastung des Balkens erfolgt im ersten Lastfall statisch, bei dem die Last zeitunabhängig wirkt. Im zweiten Lastfall wird die Last transient, also in kurzer Zeit durch eine Kraft-Zeit-Funktion aufgebracht.

\section{Modellbildung}

Die nachfolgenden Untersuchungen beziehen sich auf einen einseitig eingespannten Träger aus Aluminium mit einer Kraft von $\mathrm{F}=3.000 \mathrm{~N}$, welche am Ende des Trägers auf die Fläche der Stirnseite angreift. Die Lagerung ist dabei durch die Bindung der sechs Freiheitsgrade gekennzeichnet (Tab. 2).

\section{Konvergenzverhalten der Elemente bei statischer Belastung}

Bei der Untersuchung des Biegespannungsverlaufes in der nachstehenden Abb. 3 der Volumenelemente mit steigender Netzauflösung kann eine deutliche Annäherung an den theoretischen Wert festgestellt werden. Die Ermittlung der theoretischen Lösung für die Spannung erfolgt über die nachstehende Gleichung (1).

$$
\sigma_{b t h}=\frac{6 \cdot F \cdot l}{b \cdot h^{2}}
$$

$\begin{array}{ll}\mathrm{F} & \text { Kraft } \\ \mathrm{I} & \text { Länge } \\ \mathrm{b} & \text { Breite } \\ \mathrm{h} & \text { Höhe } \\ \sigma_{\mathrm{bth}} & \text { Biegespannung }\end{array}$

Auffallend sind die Elemente vom Typ Hexaeder mit Zwischenknoten. Diese erreichen bereits bei einer geringen Auflösung von $1 \times 3 \times 1$ eine sehr gute Annäherung an die theoretische Biegespannung. Die Auflösung beschreibt die Anzahl der finiten Elemente in den jeweiligen Koordinatenrichtungen in Höhe, Breite und Läge des Trägers. Es kann festgestellt werden, dass die Prisma- und Tetraederelemente ohne Zwischenknoten grundsätzlich ungenauere Ergebnisse liefern.

Mit steigender Auflösung der Elemente kann ein divergentes Verhalten der Biegespannung bei allen Elementtypen festgestellt werden. Die Hexaederelemente divergieren bereits in einer Auflösung von $8 \times 24 \times 1$ Elementen. Um diese Besonderheit genauer zu untersuchen, bedarf es der Darstellung der Spannungen in einem Modellschnitt. Hierzu wurde in Abb. 4 zur Verdeutlichung der Problematik eine nähere Untersuchung mit dem Elementtyp Hexaeder (quadratischer Ansatz) und einer Auflösung von $32 x$ $96 \times 1$ durchgeführt.

Es kann festgestellt werden, dass die Biegespannung an der Stelle der Einspannung, an der dem Balken alle 
Tab. 2: Untersuchungsmodell mit Parametern
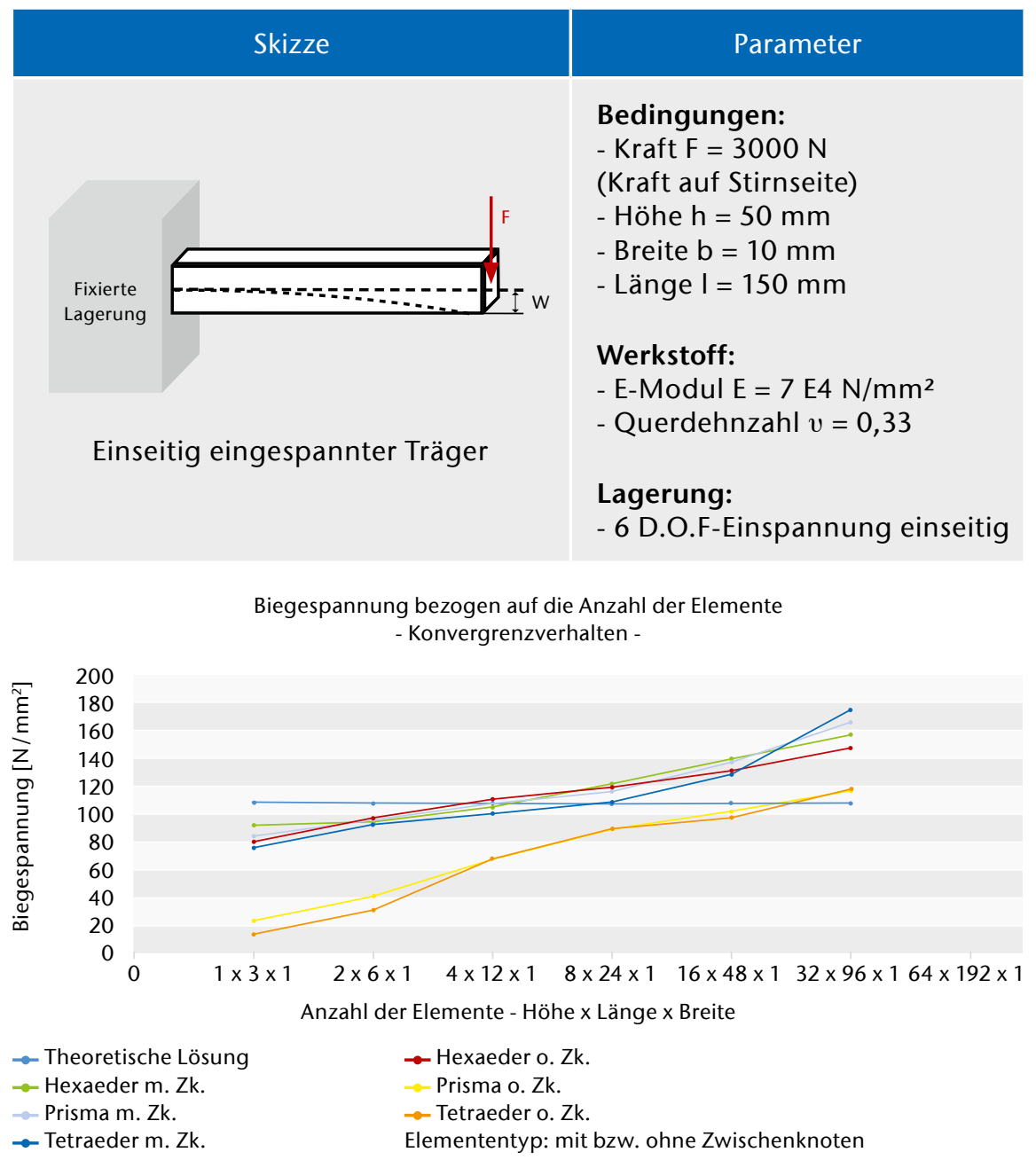

Abb. 3) Konvergenzerhalten der Biegespannung - Volumenelemente.

sechs Freiheitsgrade durch eine feste Einspannung entzogen werden, stark zunimmt. Auch mit steigender Netzdichte wird keine Konvergenz erreicht. Begründet werden kann dieser starke Spannungsanstieg durch die sich einstellende Singularität, die sich aufgrund der fixierten Lagerung im FE-System ergibt. Dabei kommt es zu sehr großen Steifigkeitssprüngen zwischen den Elementen an der Grenzfläche, was somit auch zu sehr hohen Spannungen führt. Diese Bereiche sollten aus der Konvergenzbetrachtung ausgeschlossen werden (siehe auch Gebhardt 2014). Weiterhin ist zu beachten, dass mit feiner werdendem Netz, also sehr kleinen Elementen, der numerische Fehler ansteigt.

Im nächsten Schritt erfolgt die Untersuchung zu den Elementtypen Dreieck in Abb. 5 und Tetraeder in Abb. 6. Ebenfalls wird hier die theoretische berechnete Biegespannung
$\mathrm{N} / \mathrm{mm}^{2}$ ) auf. Bei den Schalenelementen weist aber eine feinere Auflösung von $4 \times 12$ (Anzahl der Elemente in Höhe und Breite) einen geringeren relativen Fehler von 3,7 \% $\left(\sigma_{\text {bmax }}=112 \mathrm{~N} / \mathrm{mm}^{2}\right)$ auf. Bei einer Netzdichte von $2 \times 6$ wird der relative Fehler von $-4,6 \%$ $\left(\sigma_{\text {bmax }}=103 \mathrm{~N} / \mathrm{mm}^{2}\right)$ noch geringer.

In der nachfolgenden Abb. 6 ist das Verhalten der Volumenelemente vom Typ Tetraeder mit quadratischem Ansatz dargestellt. Aus der Darstellung ist ersichtlich, dass unmittelbar an der Einspannstelle die Ergebnisse bei verschiedenen Auflösungen stark schwanken und bei zu grober oder zu feiner Vernetzung zu großen Fehlerabweichungen führen.

Die geringste Abweichung erzielte die Netzdichte von $8 \times 24 \times 1$ Elementen. Das ergibt einen relativen Fehler von $1,8 \%$ bei einer Biegespannung von ob $\max =110 \mathrm{~N} / \mathrm{mm}^{2}$ gegenüber dem theoretischen berechneten Wert an der Einspannung. Wie in Abb. 6 ersichtlich ist, gibt es aber für diese Netzauflösung eine Spannungsschwankung auf $\sigma_{b}=116 \mathrm{~N} / \mathrm{mm}^{2}$ in einem Abstand von $6 \mathrm{~mm}$ vor der Einspannung und somit keinen konstant ansteigenden Spannungsverlauf.

In Auswertung der Erkenntnisse zum divergenten Spannungsverhalten an der Einspannstelle wurden weiterführende Untersuchungen durchgeführt. Dazu zählten Änderungen der Elementseitenverhältnisse bzw. das Berücksichtigen eines Radius im Bereich der Einspannung. Keine der weiteren Untersuchungen mit dem Elementtyp Tetraeder führten zu einer signifikanten Verbesserung. Vergleicht man das Konvergenzverhalten von quadratischen Schalenelementen vom Typ Dreieck mit dem der quadratischen Volumenelemente vom Typ Tetraeder, so unterliegen die Schalenelemente auch bei unterschiedlicher Netzauflösung keinen so großen Schwankungen bei der Auswertung der Biegespannung und damit des relativen Fehlers.

Aufgrund der Ungenauigkeit der Berechnungsergebnisse an der Einspannstelle bezogen auf die Biegespannung (keine Konvergenz) werden die folgenden Untersuchungen zum Nachweis der Elementgenauigkeit auf die Verformung bezogen (Abb. 7). Die Konvergenz der verschiedenen Elemente in Abhängigkeit von der 


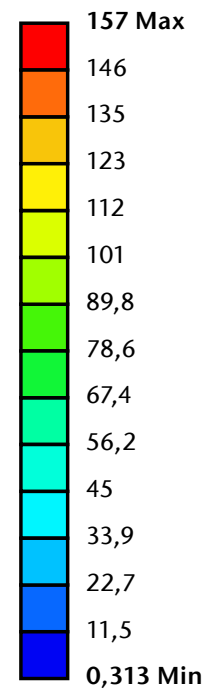

Theoretische maximale Biegespannung $=108 \mathrm{MPa}$

Abb. 4) Normalspannungsverteilung in $\mathrm{N} / \mathrm{mm}^{2}$ an der Stelle der Einspannung-Modellschnitt, feines Netz $(32 \times 96 \times 1)$

Biegespannung über der Balkenlänge - Dreieckselemente mit Zwischenknoten -

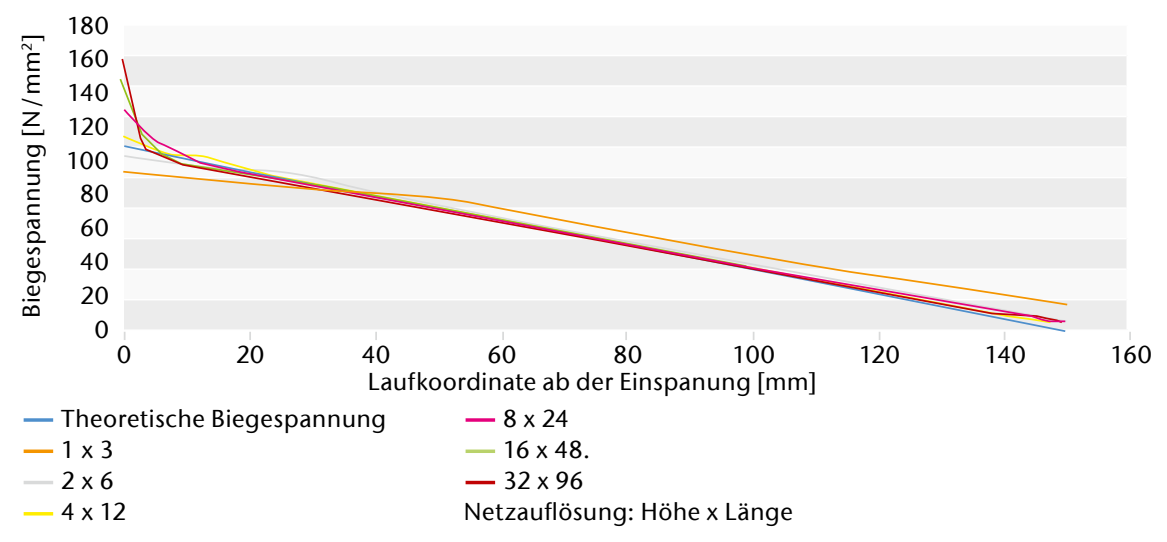

Abb. 5) Biegespannung über der Trägerlänge - Dreieckselemente mit Zwischenknoten.

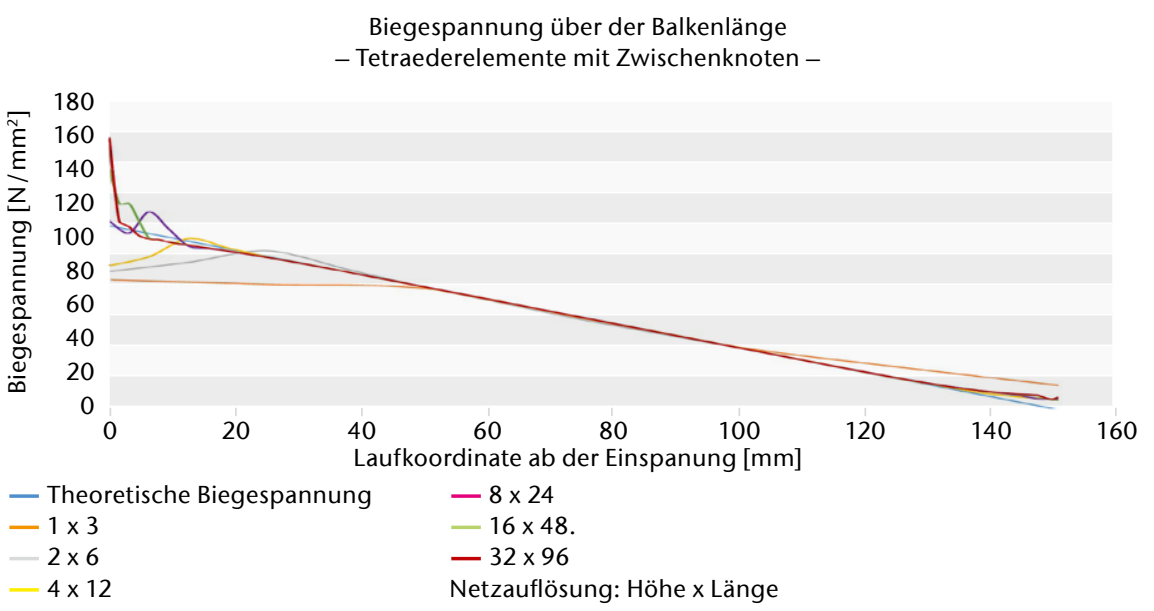

Abb. 6) Biegespannung über der Balkenlänge - Tetraederelemente mit Zwischenknoten.
Netzfeinheit ist hier eindeutig erkennbar. Die theoretisch maximal berechnete Verformung beträgt am Ende des Trägers $w=0,51 \mathrm{~mm}$ (siehe auch Tab. 2) und berücksichtigt die Durchbiegung sowie die Schubverformung. Eine Rückrechnung der Verformung auf die Biegespannungen ist an dieser Stelle des Trägers problemlos möglich. Nach den Untersuchungen wird ersichtlich, dass eine konkrete Berechnung der Biegespannung an der Einspannstelle mit der theoretischen Lösung nicht übereinstimmt. Betrachtet man hingegen den Bereich zwischen $20 \mathrm{~mm}$ und $130 \mathrm{~mm}$ vor der Einspannung, so kann ein mit der theoretischen Lösung identischer Spannungsverlauf festgestellt werden.

Wie bereits oben erwähnt sollte der Bereich der Einspannung aus der Konvergenzbetrachtung im FE-System ausgeschlossen werden. Nach einem Datenexport in ein anderes Analyseprogramm wie beispielsweise Mathcad könnte dann das Konvergenzverhalten weiterführend untersucht werden. Dabei wird die gleiche Vorgehensweise für ähnliche Probleme an anderen Bauteilen empfohlen.

\section{Genauigkeit der Elemente und Rechenzeit bei dynamischer Lastwirkung}

\section{Krafteinleitung und Dämpfung}

Zur Untersuchung des dynamischen Verhaltens des Balkens wurde die auf den Träger einwirkende Last von $\mathrm{F}=$ $3 \mathrm{kN}$ in einem Zeitintervall von 0 bis 0,00025 Sekunden festgelegt (siehe Abb. 8)

Das Antwortverhalten ist eine sich aufschwingende Struktur. Dabei wird die Schwingungsamplitude durch die Dämpfungseigenschaften des Systems fortlaufend verringert. Die Dämpfungseigenschaften eines Systems können beispielsweise durch experimentelle Untersuchungen ermittelt werden. Für den einseitig eingespannten Balken wurde eine modale Dämpfung von $\vartheta=0,05$ festgelegt. Dieser Wert orientiert sich an Empfehlungen aus der Literatur (Irretier et al. 2001). Für die Krafteinleitung und die modale Dämpfung wurde das Zeitfenster für eine dynamische Belastung von 0 Sekunden bis 0,01 Sekunden festgelegt. Der zeitliche Verlauf der Verformung 
mit und ohne Dämpfungswert ist der Abb. 9 und Abb. 10 zu entnehmen. Der Schwingungsverlauf in den Abb. 9 und 10 wurde für 1.000 Zwischenschritte berechnet. Der Zeitraum von 0 Sekunden bis zum vertikalen schwarzen Strich bei 2,5e-4 Sekunden ist der Zeitraum der Krafteinleitung. Aus dem Vergleich der oberen beiden Abbildungen ist zu erkennen, dass schon die erste Amplitude gedämpft wird.

Einfluss der Elemente und des Netzes auf die Rechenzeit

Im weiteren Verlauf der Analyse wird untersucht, wie viele Zwischenschritte benötigt werden, um hinreichend genaue Ergebnisse in Bezug zur Rechenzeit zu erhalten. Die Rechenzeit ist dabei immer die absolute Prozessorzeit des Computers, welche der Lösungsinformation im FE-System ANSYS entnommen werden kann. Bisher wurde der Einfluss der Netzauflösung auf das Konvergenzverhalten untersucht. An dieser Stelle wird nun ermittelt, welchen Einfluss die Element- und Knotenanzahl auf die Berechnungszeit haben. In der Auswertung der zeitabhängigen Belastung wird auch die Zeit der statischen Analyse zum Vergleich mit herangezogen. Beide Zeiten können jedoch nicht absolut miteinander verglichen werden, da die Zeit für die dynamische Analyse im Wesentlichen von der Zeitintervallauflösung abhängt, die es bei der statischen Analyse nicht gibt.

Der relative Verlauf der Zeiten beider Lastfälle untereinander hingegen lässt sich vergleichen. Die dynamischen Berechnungen wurden mit einer Zeitintervallauflösung von 1000 Berechnungsschritten innerhalb von 0 Sekunden bis 0,01 Sekunden durchgeführt. In der nachfolgenden Abb. 11 und Tab. 3 sind die Einflüsse der Netzauflösung am Tetraederelement dargestellt. Der Verlauf aller anderen bisher betrachtenden Elemente ist identisch und wurde der Übersichtlichkeit halber nicht mit in Abb. 11 aufgenommen.

In einer Auswertung von Abb. 11 kann festgestellt werden, dass die dynamische Berechnungszeit pro Element und Knoten ungefähr gleich ist. Aus Tab. 3 wird der Zusammenhang zwischen der Element- bzw.
Knotenanzahl und der Rechenzeit für die dynamische Analyse deutlich. So hat zum Beispiel die Auflösung $32 \times 96 \times 1$ im Vergleich zur Auflösung mit den Parametern $16 \times 48 \times 1$ in der Summe ca. 4,5-mal so viele Elemente und Knoten. Dieses Verhältnis lässt sich auch deutlich in beiden Lastfällen (statische und dynamische Analyse) erkennen.

Einfluss der zeitlichen Inkrementierung auf die Rechenzeit

Weiterführend wird auf die Zeitintervallauflösung bei der dynamischen Analyse eingegangen. Ausgewer-

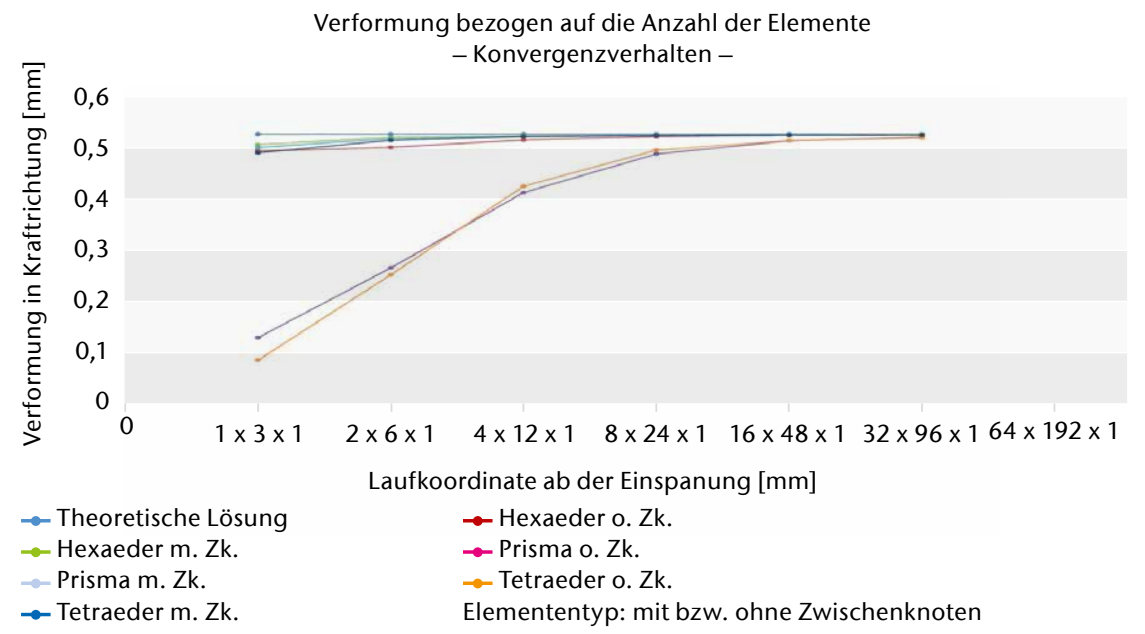

Abb. 7) Verformung über die Anzahl der Elemente - Konvergenzverhalten.

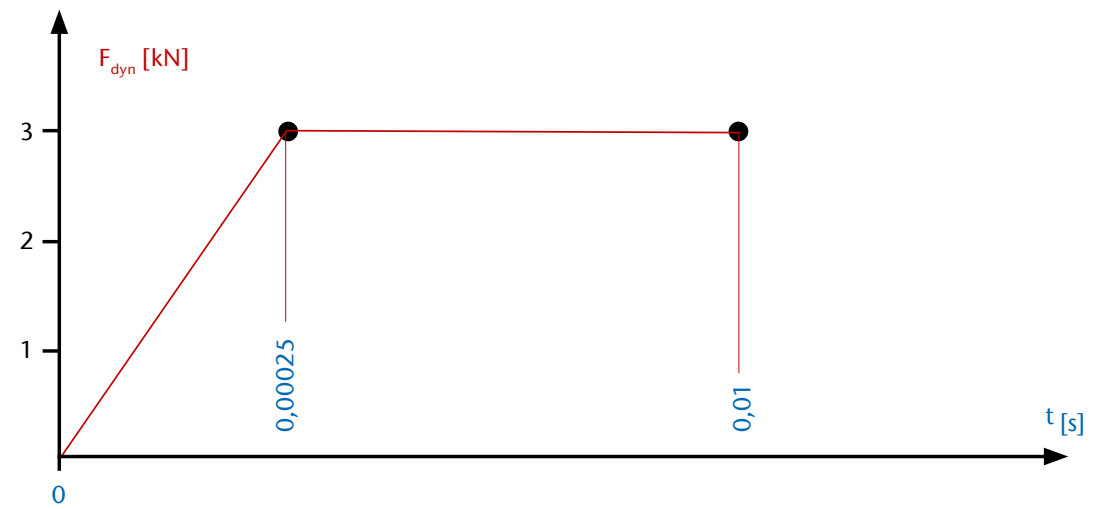

Abb. 8) Zeitabhängige Belastung des Balkens.

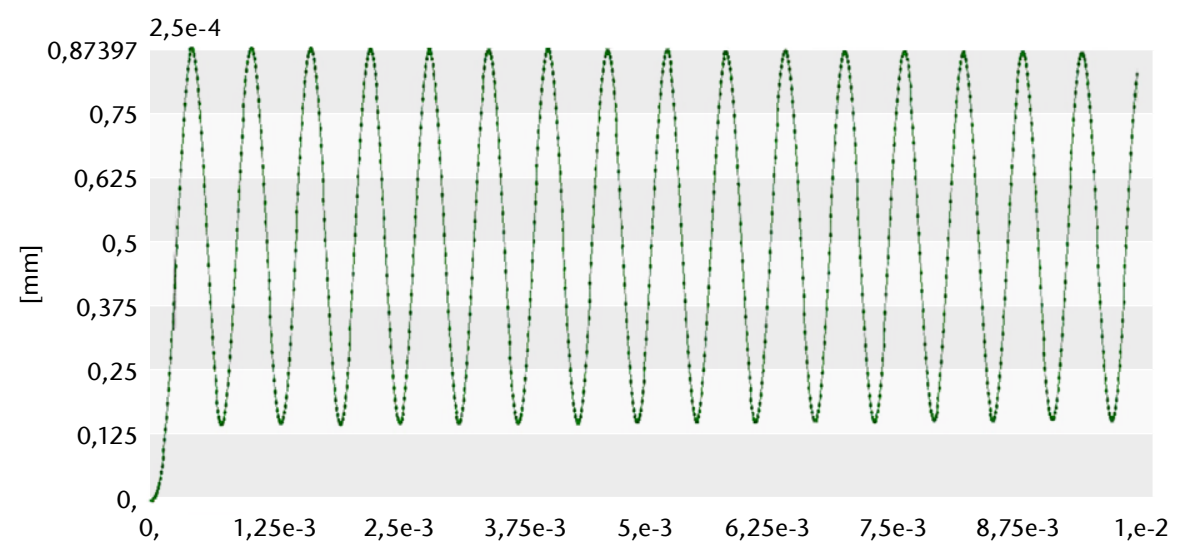

[s]

Abb. 9) Ungedämpfter Schwingungsverlauf der Verformung 
tet wurden die Verformung und die Rechenzeit in Abhängigkeit von der Anzahl der Berechnungsschritte. Die Netzauflösung und somit auch die Element- und Knotenanzahl blieb über alle Untersuchungsvarianten gleich. Die Netzauflösung war zwar bei allen Elementtypen gleich, dennoch waren die Element- und Knotenanzahlen unterschiedlich. In der Tab. 4 ist dieser Zusammenhang dargestellt.

In Tab. 4 wird auch deutlich, dass die Elemente bezüglich der Rechenzeit nicht miteinander verglichen werden können, da sich Element- und Knotenanzahl trotz gleicher Netzauflösung stark unterscheiden. Die hohe Element- und Knotenanzahl bei Tetraederelementen kann durch den unstrukturierten Netzaufbau begründet werden. Die vorgegebene Netzauflösung an den Oberflächen ließ sich nicht vollständig auf das Volumen des Trägers übertragen. In Abb. 12 sind die Elementtypen und deren Rechenzeit sowie die Verformung dargestellt. Die Abszisse zeigt die Berechnungsschritte im betrachteten Zeitbereich von 0 Sekunden bis 0,01 Sekunden auf.

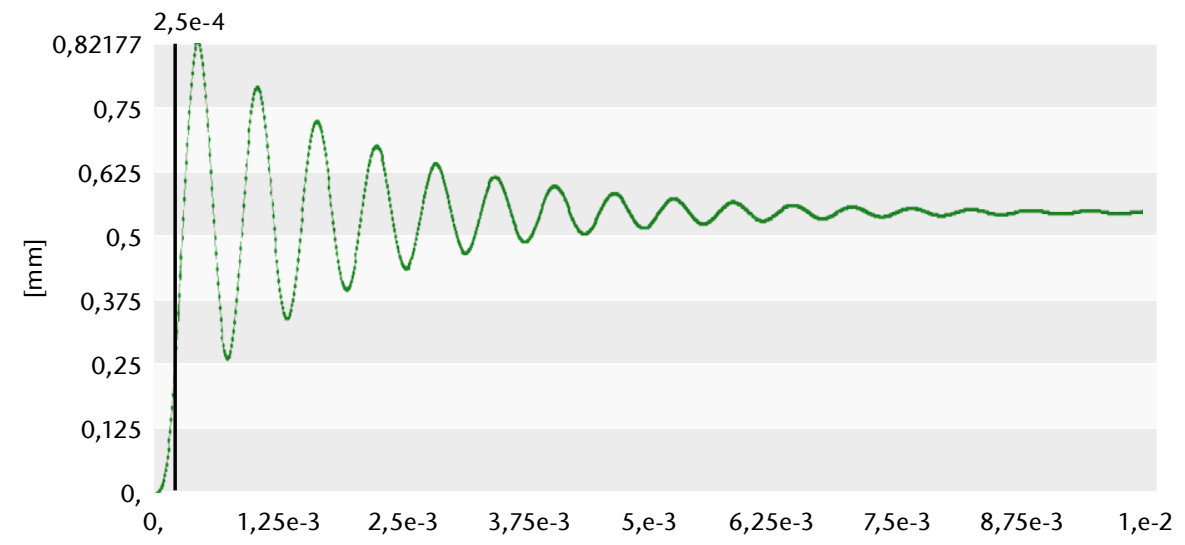

[s]

Abb. 10) Gedämpfter Schwingungsverlauf der Verformung.

Rechenzeiz bezogen auf die Anzahl der Elemente - Tetraederelemente mit Zwischenknoten -

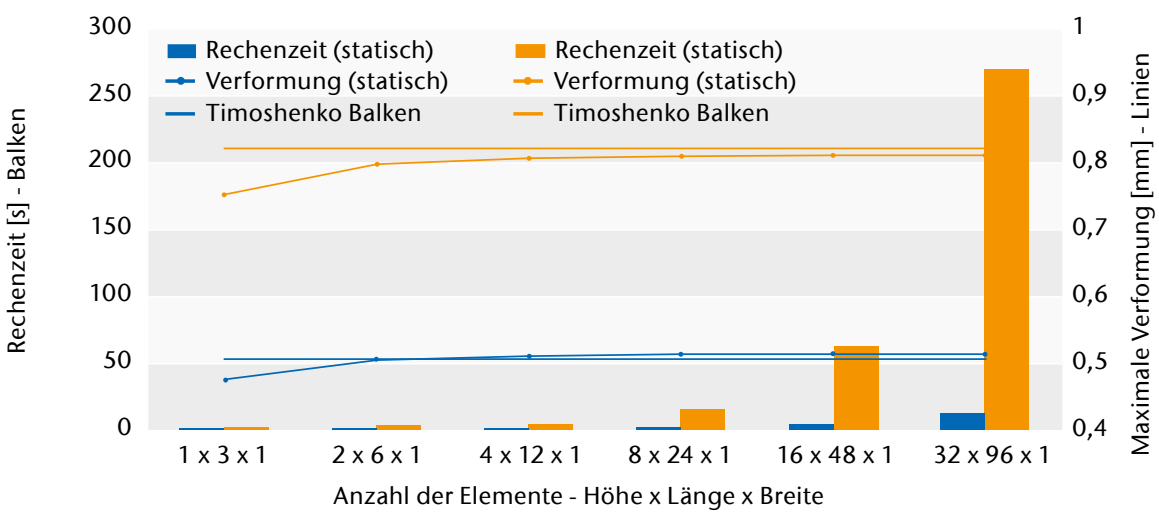

Abb. 11) Rechenzeit über die Anzahl der Elemente - Tetraeder mit Zwischenknoten.

Tab. 3: Zusammenhang zwischen Netzfeinheit und Rechenzeit für Tetraederelemente.

\begin{tabular}{|c|c|c|c|c|c|c|c|c|c|}
\hline $\begin{array}{c}\text { Element- } \\
\text { typ }\end{array}$ & $\begin{array}{c}\text { Anzahl der } \\
\text { Elemente } \\
\text { (Höhe x Länge } \\
\text { x Breite) }\end{array}$ & $\begin{array}{c}\text { Element- } \\
\text { anzahl }\end{array}$ & $\begin{array}{l}\text { Knoten- } \\
\text { anzahl }\end{array}$ & $\begin{array}{c}\text { Statische } \\
\text { Rechenzeit } \\
\text { [s] }\end{array}$ & $\begin{array}{c}\Delta \mathrm{t} \text { in } \\
{[\%] \text { zur }} \\
\text { vorherigen } \\
\text { statischen } \\
\text { Zeit }\end{array}$ & $\begin{array}{c}\Delta \mathrm{w} \text { in } \\
{[\%] \text { zur }} \\
\text { vorherigen } \\
\text { statischen } \\
\text { Verformung }\end{array}$ & $\begin{array}{l}\text { Dynami- } \\
\text { sche } \\
\text { Rechen- } \\
\text { zeit [s] }\end{array}$ & $\begin{array}{c}\Delta \mathrm{t} \text { in [\% ] } \\
\text { zur } \\
\text { vorherigen } \\
\text { dynami- } \\
\text { schen Zeit }\end{array}$ & $\begin{array}{c}\Delta \mathrm{w} \text { in [\% ] } \\
\text { zur vorhe- } \\
\text { rigen dy- } \\
\text { namischen } \\
\text { Verformung }\end{array}$ \\
\hline \multirow{6}{*}{ 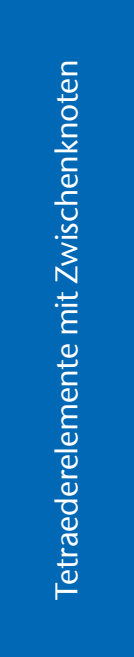 } & $1 \times 3 \times 1$ & 36 & 87 & 0,87 & - & - & 1,78 & - & - \\
\hline & $2 \times 6 \times 1$ & 114 & 251 & 0,84 & $-3,5$ & 5,36 & 2,23 & 25,4 & 5,88 \\
\hline & $4 \times 12 \times 1$ & 372 & 787 & 0,89 & 5,5 & 1,06 & 3,78 & 69 & 1,06 \\
\hline & $8 \times 24 \times 1$ & 1858 & 3437 & 1,59 & 78 & 0,43 & 14,78 & 290 & 0,43 \\
\hline & $16 \times 48 \times 1$ & 8338 & 14581 & 3,54 & 122 & 0,15 & 62,34 & 321 & 0,16 \\
\hline & $32 \times 96 \times 1$ & 36308 & 61667 & 12,84 & 262 & 0,07 & 269,79 & 332 & 0,06 \\
\hline
\end{tabular}


Aus der Abb. 12 wird ersichtlich, dass die Verformungswerte bei allen Elementtypen geringfügig voneinander abweichen, wobei die Rechenzeit unterschiedlich ist. In einer Auswertung kann festgestellt werden, dass die Rechenzeit von der Anzahl der Elemente und Knoten sowie von der Zeitauflösung abhängig ist. Die Rechenzeit steigt mit zunehmender Element- und Knotenanzahl und feiner werdender Zeitauflösung (kleinere Zeitinkremente).

Besonders hervorzuheben ist die Berechnungszeit bei Verwendung der Hexaederelemente. Sie ist bei gleichbleibender Elementezahl geringer als die der Viereckselemente, obwohl ein Hexaederelement mehr als doppelt so viel Knoten besitzt.

Um den Zeitschritt zu erkennen, bei dem die Extremwerte beispielsweise für die Spannungen und Verformungen ein Maximum ergeben, werden für die Festlegung der Zeitinkremente einer dynamischen Analyse normalerweise mehrere Kriterien herangezogen. Hierbei gelten folgende Empfehlungen (Stelzmann et al. 2008):

a) Der Zeitschritt muss klein genug sein, um die zeitabhängige Belastung gut aufzulösen. Eine diskontinuierliche Kurve sollte mit mindestens sieben Zeitschritten inkrementiert werden.

b) Die kleinste Periodendauer der Eigenfrequenzanalyse sollte in $\mathrm{min}$ destens 10 bis 20 Zeitschritten inkrementiert werden.

c) Die Geschwindigkeit der elastischen Welle sollte mit mindestens 5 bis 10 Zeitschritten inkrementiert werden (Berücksichtigung der Schallgeschwindigkeit des Materials).

Die vorliegende Untersuchung zur Berechnungszeit und zur Genauigkeit bezüglich der Verformung berücksichtigt nur die zeitliche Inkrementierung der Belastung nach Punkt a) und beschränkt sich nur auf die erste Eigenfrequenz und Eigenform.

Den Ergebnissen in Abb. 12 ist zu entnehmen, dass für alle in der Analyse verwendeten Elemente 320 Zeitschrit-
Tab. 4: Netzauflösung und Netzparameter.

\begin{tabular}{|c|c|c|c|}
\hline $\begin{array}{c}\text { Elementtyp (mit } \\
\text { Zwischenknoten) }\end{array}$ & $\begin{array}{c}\text { Anzahl der Elemente } \\
\text { (Höhe } \times \text { Länge } \times \text { Breite) }\end{array}$ & $\begin{array}{c}\text { Element- } \\
\text { typ }\end{array}$ & $\begin{array}{c}\text { Anwendung- } \\
\text { gebiet }\end{array}$ \\
\hline Timoshenko Balken & $1 \times 96 \times 1$ & 96 & 193 \\
\hline Dreieck & $32 \times 96 \times 1$ & 6144 & 12545 \\
\hline Viereck & $32 \times 96 \times 1$ & 3072 & 9473 \\
\hline Tetraeder & $32 \times 96 \times 1$ & 36308 & 61667 \\
\hline Hexaeder & $32 \times 96 \times 1$ & 3072 & 22147 \\
\hline
\end{tabular}

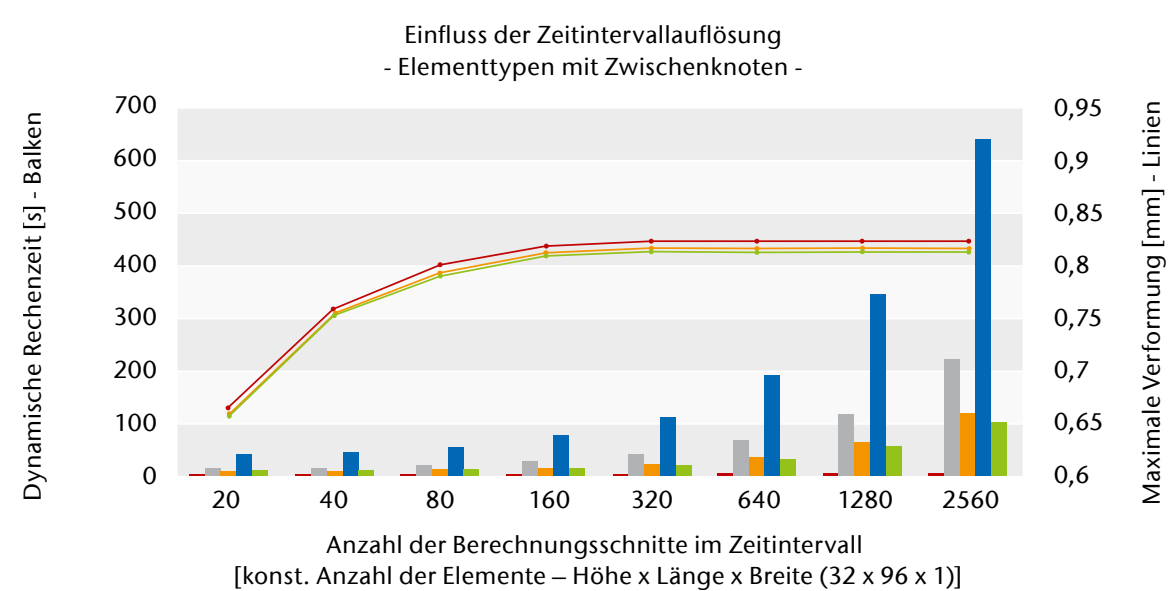

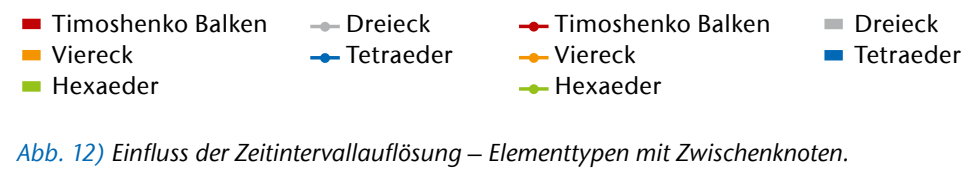

te ausreichend sind, um die maximale Verformung zu berechnen.

\section{Einfluss der Elementqualität}

Wie in vielen Veröffentlichungen publiziert (beispielsweise Klein 2015), hängt die Genauigkeit einer FE-Analyse auch von der idealen Geometrie eines finiten Elementes ab. Diese steht in einem direkten Zusammenhang mit der Interpolation der Verschiebungen durch die Ansatzfunktion innerhalb des Elementes. Ideale Formen sind bei Schalenelementen das gleichseitige Dreieck und das Quadrat, bei den Hexaederelementen der quadratische Würfel. In der Praxis sind diese idealen Formen nur bei einfachen Bauteilen zu erreichen. Hinzu kommt, dass jedes FE-Programm besondere Vernetzungsalgorithmen verwendet. Die Elementqualität kann in jedem FE-Programm geprüft wer- den. Für die Untersuchungen im ANSYS berücksichtigt die Prüfung der Elementqualität das Verhältnis der Seitenlängen einschließlich der Elementverzerrung.

Im Folgenden soll durch eine weitere Untersuchung der Nachweis erbracht werden, dass die Elementqualität Einfluss auf die Genauigkeit einer FE-Analyse hat. Hierbei wurde der Balken mit quadratischen Hexaederelementen vernetzt, um ein regelmäßiges Netz (sweepen) zu erhalten, bei dem das Verhältnis der Seitenlängen zueinander beeinflussbar ist. Dazu wurden zwei Netzauflösungen berücksichtigt.

In der nachfolgenden Abb. 13 sind die Berechnungsparameter der beiden Netzauflösungen dargestellt. Hinter der Netzauflösung auf der Abszisse befindet sich die Elementqualität als Klammerwert (1 - sehr gut; 0,16162 - schlecht). 
Einfluss der Elementqualität - Hexaederelemente mit Zwischenknoten -

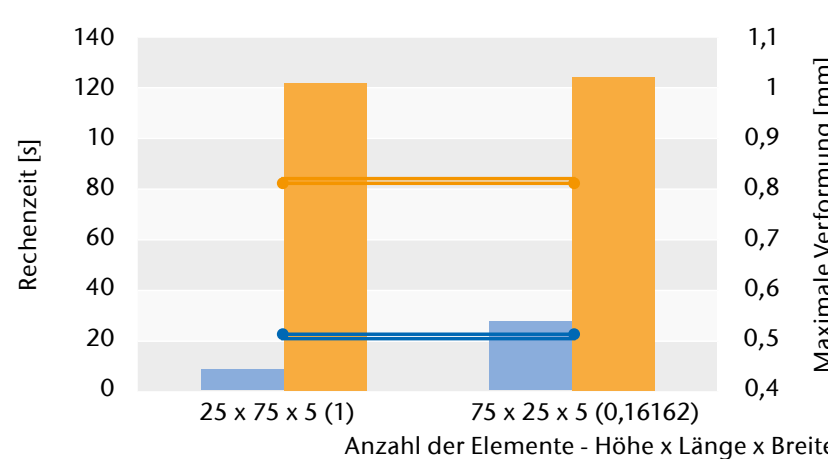

(Elementqualität)

Abb. 13) Einfluss der Elementqualität - Hexaeder mit Zwischenknoten.

Bei der Auswertung der Analyse kann festgestellt werden, dass ein starker Unterschied in der Variation der Seitenlängen eines Elementes den größten Einfluss auf die Rechenzeit der statischen Analyse hat. Die Genauigkeit der Berechnung wird bei dieser Untersuchung mit diesen Parametern nicht beeinflusst. Hierzu müssten weitere Untersuchungen vorgenommen werden.

\section{Zusammenfassung und Ausblick}

In der vorliegenden Grundlagenuntersuchung wurde die Genauigkeit bzw. das Konvergenzverhalten ausgewählter finiter Elemente bezogen auf eine einfache validierbare Problemstellung untersucht. Berücksichtigt wurden hierbei zwei Lastfälle, nämlich die statische und die dynamische Belastung eines einseitig eingespannten Trägers. Die Aufgabenstellung wurde weiterhin für linear elastisches Materialverhalten eingegrenzt.

Die Auflistung der Elementtypen und der Ergebnisse der Biegespannung hinsichtlich der Anzahl der Elemente zeigen, dass die Elemente vom Typ Hexaeder und Tetraeder mit Zwischenknoten ein sehr gutes Konvergenzverhalten aufweisen und sich der theoretischen errechneten Biegespannung annähern.

Die Stelle der Einspannung stellt durch die Randbedingung "feste Einspannung “ im Sinne des Steifigkeitsverhaltens der Elemente bezogen auf die Randfläche eine "Singularität“ dar.

Diese Bereiche sollten für die verwendeten Schalen- und Volumenelemente aus der Konvergenzbetrachtung aus- geschlossen werden. Sind genauere Ergebnisse für diesen Bereich gefordert, sollte hier mit Balkenelementen gerechnet werden bzw. sollten die Daten der FE-Analyse in einem gesonderten Analysewerkzeug wie Mathcad weiterführend untersucht werden.

Aus den Untersuchungen zur dynamischen Analyse ist zu erkennen, dass die Berechnungszeit mit der Erhöhung von Elementen und Knoten bzw. feiner werdender Inkrementierung der Zeit zunimmt. Es konnte festgestellt werden, dass ein grobes Zeitinkremment genügt, um eine ausreichende Genauigkeit der Verformung zu erhalten (320 Zeitschritte für alle untersuchten Elemente). Der ermittelte Zeitschritt berücksichtigt die ausreichende Zeitauflösung der Last und die erste Eigenmode und Eigenform. Es konnte weiterhin festgestellt werden, dass zwischen Zeitauflösung und Berechnungszeit bei kleineren Zeitinkrementen ein linearer Zusammenhang besteht. Die Elementqualität hat den größten Einfluss auf die Dauer und die Genauigkeit der statischen Analyse.

Die Ergebnisse der vorliegenden Untersuchungen haben ein hohes Potential für weiterführende Betrachtungen an anderen und komplexeren Modellen. Zunächst einmal ist zu erwarten, dass für eine Auswertung der Spannungen lineare Elementansätze sehr ungenau sind. Dieser Sachverhalt kann durch weitere Untersuchungen zu den Elementen nachgewiesen werden. Dabei ist herauszustellen, welche Elemente für eine Spannungsberechnung zweckmäßig sind. Ebenfalls kann der Einfluss der Elementqualität durch das Steifigkeitsverhalten der
Struktur mittels Modalanalyse sehr gut dargestellt werden. Hierzu wäre ein einfaches Modell, wie etwa eine allseitig eingespannte Platte, ausreichend, denn es könnte eine genaue analytische Berechnung nach Irretier (2001) erfolgen. Ein großes Potenzial liegt auch in weiteren Untersuchungen zur dynamischen Analyse,mit dem Ziel hier eine ausreichende Genauigkeit für unterschiedliche Zielgrößen (Verformung/Amplitude, Spannung oder Eigenform), bei möglichst kurzer Rechenzeit zu erhalten. In diesem Artikel wurde nur ein erster Einblick in die Problematik gegeben.

\section{KURZZEICHENVERZEICHNIS}

\begin{tabular}{|c|c|c|}
\hline Zeichen & Beschreibung & Einheit \\
\hline $\mathrm{b}$ & Breite & $\mathrm{mm}$ \\
\hline D.O.F & $\begin{array}{l}\text { Degree of Freedom - } \\
\text { Freiheitsgrade }\end{array}$ & \\
\hline $\mathrm{E}$ & Elastizitätsmodul & $\mathrm{N} / \mathrm{mm}^{2}$ \\
\hline $\mathrm{F}$ & Kraft & $N$ \\
\hline F dyn & dynamische Kraft & \\
\hline $\mathrm{FE}$ & Finite Elemente & \\
\hline FEA & Finite Elemente Analyse & \\
\hline FEM & Finite Elemente Methode & \\
\hline FE-Modell & Finite Element Modell & \\
\hline $\mathrm{h}$ & Höhe & $\mathrm{mm}$ \\
\hline I & Länge & $\mathrm{mm}$ \\
\hline $\mathrm{m}$ & Anstieg der Biegespannung & $\mathrm{N} / \mathrm{mm}^{3}$ \\
\hline o.g. & oben genannt & \\
\hline o. Zk. & ohne Zwischenknoten & \\
\hline m. Zk. & mit Zwischenkoten & \\
\hline $\mathrm{t}$ & Zeit & s \\
\hline w & $\begin{array}{l}\text { Verformung, theoretische } \\
\text { Verformung }\end{array}$ & $\mathrm{mm}$ \\
\hline$x$ & Laufkoordinate & $\mathrm{mm}$ \\
\hline$\Delta$ & Differenz & \\
\hline ob & Biegespannung & $\mathrm{N} / \mathrm{mm}^{2}$ \\
\hline ob max & maximale Biegespannung & $\mathrm{N} / \mathrm{mm}^{2}$ \\
\hline ob th & theoretische Biegespannung & $\mathrm{N} / \mathrm{mm}^{2}$ \\
\hline$\sigma_{(x)}$ & Funktion der Biegespannung & \\
\hline$v$ & Querdehnzahl & \\
\hline$\vartheta$ & $\begin{array}{l}\text { Lehr`sches Dämpfungsmaß } \\
\text { (Dämpfungsgrad) }\end{array}$ & \\
\hline
\end{tabular}




\section{LITERATUR}

Gebhardt C (2014) Praxisbuch FEM mit ANSYS Work bench. Einführung in die lineare und nichtlineare Mechanik, 2., überarbeitete Auflage. Hanser, Carl, München. ISBN: 978-3-446-43919-1

Irretier H (2001) Grundlagen der Schwingungstechnik, 1. Aufl. Vieweg, Braunschweig, Wiesbaden. ISBN: 978 3528039073

Klein B (2015) FEM. Grundlagen und Anwendungen de Finite-Element-Methode im Ma-schinen- und Fahrzeugbau, 10., verb. Aufl. Springer Vieweg, Wiesbaden. ISBN: 978-3658060534

Stelzmann U, Groth C, Müller G (2008) FEM für Praktiker. Band 2: Strukturdynamik, 5., neu bearb. Aufl. Edition expertsoft, vol 44. expert-Verl., Renningen. ISBN: 978 3816928423

Wittel FK (2010) Eine kurze Einführung in die Finite Elemente Methode.

\section{AUTOREN}

Prof. Dr.-Ing Norbert Miersch

B.Eng Robert Roßmann

B.Eng Christian Holz

Technische Hohcschule Wildau

Maschinenbau/Werkzeugkonstruktion

E-Mail für Korrespondenz:

norbert.miersch@th-wildau.de 